\title{
Correction to: Enhancing Situational Awareness and Kinesthetic Assistance for Clinicians via Augmented-Reality and Haptic Shared-Control Technologies
}

Jay Carriere, Lingbo Cheng, and Mahdi Tavakoli

\section{Correction to:}

Chapter 10 in: T. McDaniel, X. Liu (eds.), Multimedia for Accessible Human Computer Interfaces, https://doi.org/10.1007/978-3-030-70716-3_10

"Owing to an error on the part of the editor and corresponding chapter author, the author's name in the chapter opening page of chapter 'Enhancing Situational Awareness and Kinesthetic Assistance for Clinicians via Augmented-Reality and Haptic Shared-Control Technologies' was presented wrongly. The author's name has now been updated to be Mahdi Tavakoli in the chapter opening page, table of contents, and wherever applicable throughout the book." 